University of Nebraska - Lincoln

DigitalCommons@University of Nebraska - Lincoln

Publications, Agencies and Staff of the U.S.

Department of Commerce

U.S. Department of Commerce

2010

Early detection of population fragmentation using linkage disequilibrium estimation of effective population size

Phillip R. England

CSIRO Marine and Atmospheric Research and Wealth from Oceans Flagship, phillip.england@csiro.au

Gordon Luikart

University of Montana, gordon.luikart@umontana.edu

Robin Waples

NOAA, robin.waples@noaa.gov

Follow this and additional works at: https://digitalcommons.unl.edu/usdeptcommercepub

England, Phillip R.; Luikart, Gordon; and Waples, Robin, "Early detection of population fragmentation using linkage disequilibrium estimation of effective population size" (2010). Publications, Agencies and Staff of the U.S. Department of Commerce. 442.

https://digitalcommons.unl.edu/usdeptcommercepub/442

This Article is brought to you for free and open access by the U.S. Department of Commerce at DigitalCommons@University of Nebraska - Lincoln. It has been accepted for inclusion in Publications, Agencies and Staff of the U.S. Department of Commerce by an authorized administrator of DigitalCommons@University of Nebraska - Lincoln. 


\title{
Early detection of population fragmentation using linkage disequilibrium estimation of effective population size
}

\author{
Phillip R. England • Gordon Luikart • \\ Robin S. Waples
}

Received: 22 September 2009/Accepted: 13 July 2010/Published online: 24 July 2010

(C) Springer Science+Business Media B.V. 2010

\begin{abstract}
Population subdivision due to habitat loss and modification, exploitation of wild populations and altered spatial population dynamics is of increasing concern in nature. Detecting population fragmentation is therefore crucial for conservation management. Using computer simulations, we show that a single sample estimator of $N_{\mathrm{e}}$ based on linkage disequilibrium is a highly sensitive and promising indicator of recent population fragmentation and bottlenecks, even with some continued gene flow. For example, fragmentation of a panmictic population of $N_{\mathrm{e}}=1,000$ into demes of $N_{\mathrm{e}}=100$ can be detected with high probability after a single generation when estimates from this method are compared to prefragmentation estimates, given data for $\sim 20$ microsatellite loci in samples of 50 individuals. We consider a range of loci (10-40) and individuals (25-100) typical of current studies of natural populations and show that increasing the number of loci gives nearly the same increase in precision as increasing the number of individuals sampled. We also evaluated effects of incomplete fragmentation and found this $N_{\mathrm{e}^{-}}$
\end{abstract}

\footnotetext{
P. R. England $(\varangle)$

CSIRO Marine and Atmospheric Research and Wealth from Oceans Flagship, GPO Box 1538, Hobart, TAS 7001, Australia e-mail: phillip.england@csiro.au

G. Luikart

Flathead Lake Biological Station (FLBS), University

of Montana, Polson, MT 59860, USA

G. Luikart

Centro de Investigação em Biodiversidade e Recursos Genéticos (CIBIO-UP), Universidade do Porto, 4485-661 Vairão, Portugal
}

R. S. Waples

NOAA Fisheries, Northwest Fisheries Science Centre,

2725 Montlake Blvd. East, Seattle, WA 98112, USA reduction signal is still apparent in the presence of considerable migration $(m \sim 0.10-0.25)$. Single-sample genetic estimates of $N_{\mathrm{e}}$ thus show considerable promise for early detection of population fragmentation and decline.

Keywords $N_{\mathrm{e}}$. Effective population size . Linkage disequilibrium · Fragmentation · Bottleneck · Connectivity $\cdot$ Monitoring $\cdot$ Conservation

\section{Introduction}

The effective population size $\left(N_{\mathrm{e}}\right)$ is one of the most important parameters in conservation and evolutionary genetics. It influences key evolutionary processes such as the rate of loss of neutral genetic variation due to drift, the rate of accumulation of deleterious mutations and the ability of a population to respond to natural and humaninduced selection. Reduction of population size and $N_{\mathrm{e}}$ from fragmentation and decline is increasingly threatening populations world wide due to habitat loss and modification, exploitation and altered spatial population dynamics (Frankham et al. 2002; Allendorf and Luikart 2006). Detecting population fragmentation is therefore crucial for conservation management.

Reduction of local $N_{\mathrm{e}}$ due to subdivision of a oncewidespread population into demes or sub-populations has the potential to be exploited as an indicator of population fragmentation, yet this possibility has not been previously investigated. As the need for monitoring tools for management of threatened populations increases, so does the imperative to develop genetic methods that might be deployed rapidly and efficiently.

Detecting change (e.g. in $N_{e}$ ) normally requires two samples separated in time. The lack of historical samples 
for many (perhaps most) natural populations and/or lengthy generation intervals limits the number of situations where the temporal approach can be adopted. However, detection of small contemporary $N_{\mathrm{e}}$ from single samples has the potential to alert us to cryptic recent (local) population decline or fragmentation, especially in situations where other evidence suggests the population should be large and interconnected.

Unfortunately, $N_{\mathrm{e}}$ is notoriously difficult to estimate in wild populations because it is hard to measure all the demographic factors that influence it (Frankham 2005). Estimation from genetic data offers a convenient and popular alternative. A variety of methods is available (Schwartz et al. 1998; Wang 2005; Leberg 2005; Ovenden et al. 2007), including one that estimates $N_{\mathrm{e}}$ from linkage disequilibrium (LD) i.e., gametic disequilibrium existing in a single sample of individuals (Hill 1981; England et al. 2006; Waples 2006). Being able to estimate $N_{\mathrm{e}}$ from a single sample makes the LD method and several related methods (Tallmon et al. 2008; Vitalis and Couvet 2001; Cornuet and Luikart 1996; Nomura 2008; Wang 2009) potentially important tools for species for which historical samples and knowledge are lacking, but where assessment and monitoring of genetic parameters are considered high priorities.

We present here an application of the LD method that has potential as a tool for detection of the increasingly widespread and threatening ecological phenomenon of population fragmentation and subdivision (reviewed in Young and Clark 2000). This approach explores our initial observation that when a panmictic population is subdivided into demes (as increasingly occurs in nature following habitat fragmentation, altered migration rates and spatially uneven exploitation), the decline in local $N_{\mathrm{e}}$ is almost instantaneous. This makes the estimator a potentially more sensitive indicator of recent subdivision than other genetic changes such as loss of genetic diversity or increased differentiation among demes (e.g., $F_{\text {st }}$ ). We used individualbased simulations to model population subdivision over a range of scenarios of complete and incomplete subdivision where interdeme migration continued to varying extents.

Situations where cryptic population fragmentation might occur and where the detection of local declines in $N_{e}$ might alert managers to its occurrence include species with ranges spanning increasingly fragmented forest or grassland, aquatic habitats, coastal marine species with patchy distributions vulnerable to isolation, or any species who's connectivity could become reduced (e.g. due to humaninduced landscape change, over harvest, pollution, disease, or competition from invasive taxa in part of the species' range).

Other single sample methods for estimating $N_{\mathrm{e}}$ (e.g. Tallmon et al. 2008; Wang 2009) are also promising tools for the detection of fragmentation scenarios, and they may have similar or higher power than the LD method under certain circumstances, however these are computationally very intensive and do not lend themselves to a large-scale simulation-based investigation such as that presented here. Of course, the ability to infer a decline in the local $N_{\mathrm{e}}$ relies upon being able to compare it with a reasonable expectation that the former populations size was large and interconnected, based on other information or earlier $N_{\mathrm{e}}$ estimates.

\section{Methods}

We used the program EASYPOP 1.7.1 (Balloux 2001) to simulate populations with specified $N_{\mathrm{e}}$. EAsYPoP is a stochastic, individual-based Wright-Fisher simulation program that models Mendelian inheritance. In simulations of this type, the mean realized $N_{e}$ approximates the population census size $N_{c}$ when the sex ratio is one. We simulated demes over a range of local effective sizes likely to be of concern to conservation biologists $\left(N_{\mathrm{e}}=20-100\right)$ and used sample sizes (25-100) and numbers of loci (10-40) that are typical of most contemporary studies of natural populations. Simulations were initiated with 10 alleles per locus at equal frequency and a mutation rate of $5 \times 10^{-4}$ to typify microsatellite diversity (Estoup and Angers 1998). To simulate effective sizes lower than census size in EAsYPoP, we simulated distorted sex ratios according to the formula $N_{\mathrm{e}}=4 N_{m} N_{f} /\left(N_{f}+N_{m}\right) \quad$ (Wright 1931) that gave the required $N / N_{\mathrm{e}}$ ratio. We used this approach to allow us to keep the census size constant for the entire metapopulation across all evaluations while allowing $N_{\mathrm{e}}$ to vary.

Simulations were initiated by randomly choosing multilocus genotypes for 1,000 diploid individuals, with each of 10 alleles having an equal probability of being assigned (maximal diversity option). The initial metapopulation consisted of 10 demes $(N=100)$ with a migration rate ( $m=0.9$; meaning that individuals migrated to another deme with $90 \%$ probability) that equates to global panmixia. Parents mate locally and produce offspring, which then either migrate or not, before they reproduce and die. At generation $10, m$ was reduced to $0,0.01,0.05$ or 0.25 , creating 10 more or less isolated demes of 100 individuals each. After fragmentation, genetic parameters were monitored in each deme for up to 10 generations.

Prior to the reduction in migration, demes are in effect imaginary, as the population as a whole is panmictic. An analogy might be a patchily distributed species with high gene flow among patches that suddenly decreases in response to a perturbation. Under these conditions, local sampling of adults initially will be equivalent to sampling from the entire metapopulation, but as gene flow becomes 
restricted local sampling will increasingly yield an estimate related to the local $N_{\mathrm{e}}$.

$N_{\mathrm{e}}$ was calculated using the software $\mathrm{LD} N_{\mathrm{e}}$ (Waples and Do 2008) which implements an estimator of inbreeding effective population size (Waples 2006) that uses Weir's (1979) unbiased estimator of Burrows' $\Delta$. $\mathrm{LDN}_{e}$ performs well with skewed sex ratios such as those used to simulate reduced $N_{\mathrm{e}} / N$ ratios in our study (Waples 2006), as well as with overdispersed variance in reproductive success (not considered here, but probably ubiquitous in nature). We used the random mating model option, a critical allele frequency value of 0.02 (alleles with frequency $<0.02$ were excluded; Waples and Do $(2008,2010)$ indicated that this helps maximize precision without creating undue bias from use of rare alleles), and recorded parametric and jacknife $95 \%$ confidence intervals. $\hat{N}_{e}$ and its confidence interval were estimated at generation $0,1,2,3,5$ and 10 in 100 independent replicate demes (one deme per replicate). We recorded the harmonic means of the $N_{e}$ estimates and the proportion of simulations of each scenario that excluded the "nominal" or "true" $N_{e}$ on the high or low side.

\section{Results and discussion}

In all simulated scenarios, linkage disequilibrium-based estimates of $N_{e}\left(\hat{N}_{e}\right)$ declined sharply immediately after subdivision and approached the nominal $N_{e}$ rapidly (Fig. 1a, b). Confidence intervals of the estimates of the unfragmented population do not overlap with those from G1 and later (Table 1). In agreement with results presented by Waples $(2005,2006)$, estimates were slightly biased upwards immediately after subdivision, but this bias was negligible by the third generation. This suggests relatively high power to detect population subdivision or reduced gene flow using this $N_{\mathrm{e}}$ estimator, even immediately after (i.e. one generation) the subdivision has occurred, provided the population fragments are not too large (e.g. $N_{e} \gg 500$ ) and provided adequate data are available.

The confidence intervals (CI) on estimates from demes of size 100 fell entirely below the original population $N_{e}$ of 1,000 at generation one (G1) and every subsequent generation in $100 \%$ of cases, when using samples of 100 . This was also true in samples of 50 with 20-40 loci (Table 2). Using fewer loci and individuals lowers precision and therefore reduces the power to detect local population decline. This is indicated by decreased proportions of CIs falling below, i.e. excluding 200 (or 1,000) when the sample size or number of loci was decreased (Table 2). For instance, using 20 loci and samples of 100 individuals, the upper CI boundary was less than 200 at G1 in $96 \%$ of simulated populations and in $73 \%$ of simulations using 10 loci (Table 2). This demonstrates high power to quickly
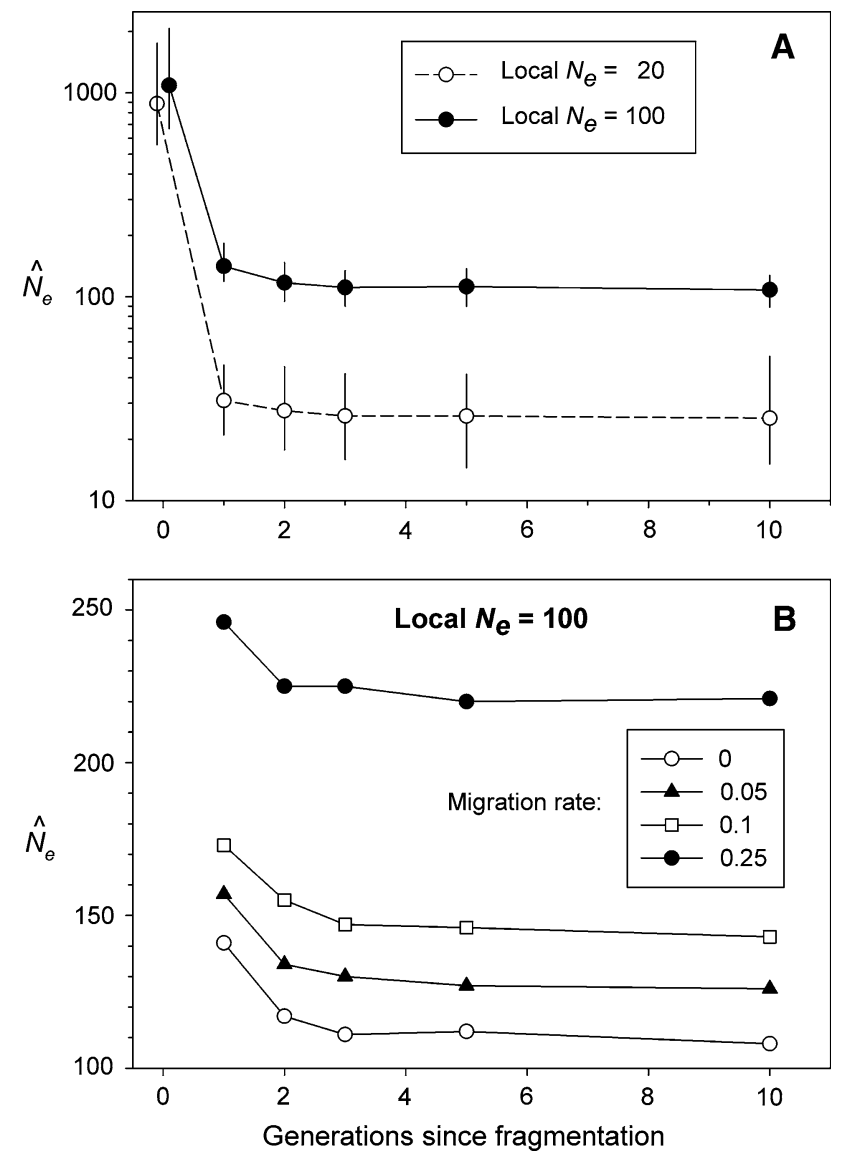

Fig. 1 a Harmonic mean $\hat{N}_{e}$ (with 2.5th and 97.5th percentiles of the distribution) of replicate simulations in the unfragmented metapopulation $(\mathrm{G} 0)$ and the ten generations after fragmentation of a single large population $\left(N_{e}=1,000\right)$ into isolated demes of size 100 or 20; b as above, but with incomplete fragmentation (continued migration among demes of $N=100$ ) and the case for no migration included for comparison. Estimates were obtained by sampling the entire deme

detect a sudden reduction in the original population size. We used $N_{\mathrm{e}} 200$ as a yardstick because CIs that lie below this boundary indicate the new estimate is convincingly less than $20 \%$ of original population size and no more than twice the new deme size.

Increasing the sample size improved precision and accuracy slightly more than the same proportional increase in the number of loci. For example, with 10 loci and a sample of 25 , only $37 \%$ of parametric CIs excluded the original population size 1,000 after one generation (Table 2). Under these conditions, doubling the number of loci improved precision less than doubling the sample size (73\% vs. $92 \%$ of CIs excluded the original $N$ ). With a sample of 50 and using 20 loci the upper CI boundary excluded 200 at G1 in $49 \%$ of simulations. As with the previous example, doubling the number of loci improved precision less than doubling the sample size $(69 \%$ CIs excluded 200 with twice the loci vs. $96 \%$ by doubling the 
Table 1 Harmonic mean $\hat{N}_{e}$ (with 2.5th and 97.5th percentiles of the distribution of replicate simulations) and percentiles of the distributions of lower and upper parametric confidence interval boundaries for up to 10 generations post subdivision in isolated demes of effective size 100 and 20 using all individuals from the deme as samples

\begin{tabular}{|c|c|c|c|c|c|c|c|c|c|c|c|}
\hline \multirow[t]{2}{*}{$\hat{N}_{e}$} & & \multicolumn{5}{|c|}{ Percentiles of lower CI boundary } & \multicolumn{5}{|c|}{ Percentiles of upper CI boundary } \\
\hline & & 5 & 20 & 50 & 80 & 95 & 5 & 20 & 50 & 80 & 95 \\
\hline \multicolumn{12}{|c|}{$N_{e}=100$} \\
\hline G0 & $1086(6682068)$ & 558 & 675 & 781 & 971 & 1111 & 932 & 1298 & 1740 & 3031 & 4910 \\
\hline G1 & $141(119183)$ & 109 & 115 & 124 & 139 & 141 & 135 & 145 & 158 & 182 & 186 \\
\hline $\mathrm{G} 2$ & 117 (95 147) & 91 & 97 & 105 & 114 & 122 & 110 & 119 & 130 & 143 & 155 \\
\hline G3 & $111(90134)$ & 88 & 93 & 99 & 110 & 117 & 106 & 114 & 122 & 138 & 148 \\
\hline G5 & 112 (90 137) & 81 & 93 & 101 & 108 & 120 & 99 & 113 & 124 & 135 & 152 \\
\hline G10 & 108 (89 127) & 84 & 91 & 98 & 105 & 111 & 102 & 112 & 122 & 132 & 141 \\
\hline \multicolumn{12}{|c|}{$N_{e}=20$} \\
\hline G0 & $884(557$ 1251) & 475 & 547 & 676 & 849 & 1006 & 725 & 903 & 1301 & 2088 & 3390 \\
\hline G1 & $30.94(2146)$ & 19 & 22 & 26 & 31 & 36 & 26 & 31 & 38 & 48 & 58 \\
\hline G2 & $27.55(1845)$ & 16 & 20 & 24 & 30 & 33 & 22 & 28 & 35 & 47 & 56 \\
\hline G3 & $25.98(1642)$ & 15 & 19 & 24 & 26 & 29 & 21 & 28 & 36 & 40 & 50 \\
\hline G5 & $25.96(1442)$ & 14 & 19 & 23 & 25 & 30 & 21 & 29 & 37 & 45 & 63 \\
\hline G10 & $25.33(1551)$ & 12 & 16 & 19 & 24 & 32 & 21 & 28 & 39 & 57 & 98 \\
\hline
\end{tabular}

Table 2 Harmonic mean of $\hat{N}_{e}$ for 1-10 generations post subdivision in subdivided populations of deme size 100 (computed from 100 independent replicates) and percentages of parametric and jacknife 95\% confidence intervals on $\hat{N}_{e}$ that: were entirely above the modelled deme size of $100(>100)$; entirely below $200(<200)$; entirely below $1,000(<1,000)$

Loci Sample size $\hat{N}_{e} \frac{\text { Parametric CIs }}{>100<200<1000} \frac{\text { Jacknife CIs }}{>100<200<1000}$

G1 (Generation 1)

\begin{tabular}{rrrrrrrrr}
10 & 100 & 142 & 88 & 73 & 100 & 92 & 74 & 100 \\
10 & 50 & 150 & 50 & 21 & 92 & 47 & 29 & 94 \\
10 & 25 & 141 & 10 & 10 & 37 & 20 & 15 & 50 \\
20 & 100 & 141 & 99 & 96 & 100 & 99 & 96 & 100 \\
20 & 50 & 153 & 90 & 49 & 100 & 91 & 52 & 100 \\
20 & 25 & 117 & 63 & 15 & 73 & 71 & 23 & 80 \\
40 & 100 & 141 & 100 & 100 & 100 & 100 & 100 & 100 \\
40 & 50 & 151 & 100 & 69 & 100 & 100 & 73 & 100 \\
40 & 25 & 165 & 86 & 39 & 96 & 89 & 44 & 98 \\
G2 & & & & & & & & \\
20 & 100 & 117 & 73 & 100 & 100 & 72 & 100 & 100 \\
G3 & & & & & & & & \\
20 & 100 & 111 & 46 & 100 & 100 & 48 & 100 & 100 \\
G5 & & & & & & & & \\
20 & 100 & 112 & 51 & 100 & 100 & 48 & 100 & 100 \\
G10 & & & & & & & & \\
20 & 100 & 108 & 37 & 100 & 100 & 32 & 100 & 100 \\
\hline
\end{tabular}

sample size). Based on Hill's (1981) approximation to the variance of $\hat{N}_{e}$ for the LD method, Waples and Do (2010) concluded that, in general, doubling samples of individuals or loci should have roughly the same effect on precision. In this case, we believe that doubling sample size was slightly more effective because doing so also increased the mean number of alleles that could be used in the analysis; in contrast, the analysis by Waples and Do assumed the number of alleles per locus remained fixed when sample size changed. Another likely contributing factor is that the Waples and Do analysis approximation assumes that each locus pair is independent, which is not entirely true (Hill 1981; Waples 2006). As a consequence, the gains in precision to be expected from doubling the number of loci are not as large as their analysis would suggest.

Similar effects were observed in smaller demes. For example, when deme effective size was 20 , the upper CI was less than 59 at G1 in $95 \%$ of simulations (95th percentile of upper confidence interval $=58$, Table 1 ) indicating high power to detect reduced population size in population fragments. Lower CIs were above the nominal deme size in $80 \%$ of simulations at G1 (20th percentile of lower $\mathrm{CI}=22$, Table 1 ) but by $\mathrm{G} 2$ the 20th percentile encompassed the nominal deme size.

Varying the $N_{\mathrm{e}} / N$ ratio with $N_{e}$ held constant had no noticeable effect. For example, when $N_{\mathrm{e}}$ was substantially smaller than $N_{\mathrm{c}}\left(N_{\mathrm{e}}=20 ; N_{\mathrm{c}}=100\right) \hat{N}_{e}$ and its CIs were similar to those from simulations using $N_{\mathrm{e}}=N_{\mathrm{c}}=20$ when the same sample sizes were used (data not shown). This result highlights the importance of monitoring effective population size as well as (or instead of) census size, as apparently large populations can have cryptically low effective sizes of conservation genetic concern. 
The ability of this method to detect fragmentation is surprisingly robust to limited migration among demes that continues after fragmentation (Fig. 1b). With residual migration at $m=0.25, \mathrm{LDN}_{e}$ settles down to $\sim 220$ after 3 generations, reflecting the fact that the number of parents contributing to a sample is larger than the local effective size. With migration of $5 \%$, estimates are elevated by about 10-20\% over those from completely isolated demes, but even at G1 are dramatically reduced compared to the unfragmented population size. No upper $95 \%$ confidence intervals on $\mathrm{LDN}_{e}$ with $m=0.25$ using 20 loci encompassed 1,000 at $\mathrm{G} 1$ or any subsequent generation. The effect of migration among demes on $\mathrm{LDN}_{e}$ is explored further in a companion paper (England and Waples in prep).

Bottleneck tests, similar to $\mathrm{LDN}_{e}$, use a single sample of individuals to detect recent population declines (Cornuet and Luikart 1996) and they appear to provide similar power to detect recent population declines or fragmentation (Luikart unpublished data). However, unlike $\operatorname{LDN}_{e}$, bottleneck tests do not provide a point estimate of $N_{\mathrm{e}}$, but rather detect only a recent change in $N_{\mathrm{e}}$ (Luikart et al. 1999). Future research is needed to compare power of bottleneck tests and one-sample estimators of $N_{e}$ for monitoring to detect population declines.

LD-based estimates of $N_{e}$ were more sensitive to fragmentation in the generations immediately after it occured than typical genetic measures of subdivision such as loss of expected heterozygosity $\left(H_{e}\right)$ and $F_{\text {st }}$. For example, for the scenario simulated extensively in this paper (subdivision of a population of 1,000 individuals into 10 isolated demes of 100), $F_{\text {st }}$ had increased by 0.0045 and $H_{e}$ had declined by $0.5 \%$ at $\mathrm{G} 1$ compared to a reduction of $86 \%$ in $\operatorname{LD} \hat{N}_{e}$. At G10 $F_{\text {st }}$ had increased to 0.044 and $H_{e}$ had declined by $4.8 \%$ whereas $\operatorname{LDN}_{e}$ had changed by $92 \%$. Zartman et al. (2006) found a similar result in an empirical study of fragmentation in the Amazonian liverwort Radula flaccida: strong evidence for linkage disequilibrium but little indication of population genetic structure or loss of genetic diversity.

It is easy to conceive of modern scenarios that might cause sudden population subdivision of concern in conservation e.g. patchy deforestation, road construction, altered agricultural practices, shifting spatial patterns of population exploitation, and reduced terrestrial hydrology and marine hydrodynamics from climate change. In many of these cases, effects on population connectivity could be cryptic and therefore easily overlooked and painstaking to measure. The $\mathrm{LD} \hat{N}_{e}$ method offers a robust, and relatively rapid (i.e. requiring only a single generation sample) way of detecting the effects of subdivision.

Waples and Do (2010) suggest that unless temporal samples are separated by many generations, single-sample methods of estimating $N_{\mathrm{e}}$ will generally be more precise than the temporal method. It will be important to evaluate the relative power of LD and other single-sample $N_{e}$ estimation methods to act as tools for monitoring the subdivision effect we describe here. It is surprising greater effort has not been invested in genetic methods for detection of population subdivision, given the increasing prevalence of and extinction threat posed by this phenomenon in the wild.

A potential limitation of the approach is the assumption that each sample comes from a single deme. If putative subpopulations cannot reliably be identified $a$ priori, or the causes or boundaries of subdivision are unknown, this might be difficult to ensure. If samples comprise individuals from multiple fragmenting demes, estimates could be biased high because more breeders have contributed to the sample, potentially obscuring the subdivision signal. Alternatively, if highly divergent demes are mistakenly pooled, estimates could be biased low due to excessive mixture LD (Nei and Li 1973). Other potentially less problematic assumptions include that generations are discrete, the loci are unlinked, and the alleles studied are selectively neutral.

Future research should assess the effects of violating these assumptions of the LD method (and the other $N_{e}$ estimators) on their utility for detecting population fragmentation. If the method is to be contemplated as a monitoring tool, spatial sampling design and analyses may need to anticipate likely or possible deme boundaries.

\section{Conclusions}

We have demonstrated that monitoring $N_{\mathrm{e}}$ from linkage disequilibrium can be a sensitive early indicator of subdivision under two realistic scenarios. First, if at least two samples are available that span a fragmentation event, comparison of the two estimates can provide a powerful means of detecting declines in $N_{\mathrm{e}}$. In contrast, the temporal method provides a single estimate of $N_{\mathrm{e}}$ across the generations sampled so cannot detect a trend without at least 3 samples. Second, if only a single (post-fragmentation) sample is available, the LD method (or another single sample method) can still function as a red flag to warn when local population size has declined to a range that is of conservation concern. If fragmentation produces relatively small demes $\left(N_{e}\right.$ around 100), power to detect fragmentation can be very high $(>0.80)$ immediately after fragmentation. Incomplete subdivision or "leakage of migrants" is likely to be a common outcome of partial habitat fragmentation. Encouragingly, our results indicate that even substantial continued migration does not necessarily obscure the signal of subdivision on $\operatorname{LDN}_{\mathrm{e}}$, potentially 
broadening the method's usefulness. This topic is further examined in another paper (England and Waples in prep.). The increasing ease with which large marker sets can be genotyped, along with noninvasive sampling and the availability of software to generate these estimates, makes this a potentially important tool for early warning and monitoring for population bottlenecks, subdivision and habitat fragmentation.

Acknowledgments PRE was assisted by an Australian Academy of Sciences Visit to North America Fellowship. GL was partially supported by a grant to FLBS from the Walton Family Foundation and by the Portuguese-American Foundation for Development, CIBIO, and UP. We thank Fred Allendorf for discussions and hosting the visit to his lab by PRE.

\section{References}

Allendorf FW, Luikart G (2006) Conservation and the genetics of populations. Wiley Blackwell, Oxford

Balloux F (2001) EasyPop (version 1.7): a computer program for population genetics simulations. J Hered 92:301-302

Cornuet J-M, Luikart G (1996) Description and power analysis of two tests for detecting recent population bottlenecks from allele frequency data. Genetics 144:2001-2014

England PR, Cornuet J-M, Berthier P, Tallmon DA, Luikart G (2006) Estimating effective population size from linkage disequilibrium: severe bias using small samples. Conserv Genet 7:303-308

Estoup A, Angers B (1998) Microsatellites and minisatellites for molecular ecology. In: Carvalho G (ed) Advances in molecular ecology. IOS Press, Amsterdam, pp 55-86

Frankham R (2005) Genetics and extinction. Biol Conserv 126: $131-140$

Frankham R, Ballou JD, Briscoe DA (2002) Introduction to conservation genetics. Cambridge University Press, Cambridge

Hill WG (1981) Estimation of effective population size from data on linkage disequilibrium. Genet Res Camb 38:209-216

Leberg P (2005) Genetic approaches for estimating the effective size of populations. J Wildl Manag 69:1385-1399

Luikart G, Cornuet J-M, Allendorf FW (1999) Temporal changes in allele frequencies provide estimates of population bottleneck size. Conserv Biol 13:523-530

Nei M, Li W-H (1973) Linkage disequilibrium in subdivided populations. Genetics 75:213-219
Nomura T (2008) Estimation of effective number of breeders from molecular coancestry of single cohort sample. Evol Appl $1: 462-474$

Ovenden JR, Peel D, Street R, Courtney AJ, Hoyle SD, Peel S, Podlich H (2007) The genetic effective and adult census sixe of an Australian population of tiger prawns (Penaeus esculentus). Mol Ecol 16:127-138

Schwartz MK, Tallmon DA, Luikart G (1998) Review of DNA-based census and effective population size estimators. Anim Conserv 1:293-299

Tallmon DA, Koyuk A, Luikart G, Beaumont MA (2008) OneSamp: a program to estimate effective population size using approximate Bayesian computation. Mol Ecol Resour 8:299-301

Vitalis R, Couvet D (2001) Estimation of effective population size and migration rate 22 from one- and two-locus identity measures. Genetics 157:911-925

Wang J (2005) Estimation of effective population sizes from data on genetic markers. Philos Trans R Soc B 360:1395-1409

Wang J (2009) A new method for estimating effective population size from a single sample of multilocus genotypes. Mol Ecol 18: $2148-2164$

Waples RS (2005) Genetic estimates of contemporary effective population size: to what time periods do the estimates apply? Mol Ecol 14:3335-3352

Waples RS (2006) A bias correction for estimates of effective population size based on linkage disequilibrium at unlinked gene loci. Conserv Genet 7(2):167-184

Waples RS, Do C (2008) $L D N E$ : a program for estimating effective population size from data on linkage disequilibrium. Mol Ecol Resour 8:753-756

Waples RS, Do C (2010) Linkage disequilibrium estimates of contemporary $N_{e}$ using highly variable genetic markers: a largely untapped resource for applied conservation and evolution. Evol Appl 3:244-262

Weir BS (1979) Inferences about linkage disequilibrium. Biometrics $35: 235-254$

Wright S (1931) Evolution in Mendelian populations. Genetics 16:97-159

Young AG, Clark GM (eds) (2000) Genetics demography and viability of fragmented populations. Cambridge University Press, England

Zartman CE, McDaniel SF, Shaw J (2006) Experimental habitat fragmentation increases linkage disequilibrium but does not affect genetic diversity or population structure in the Amazonian liverwort Radula flaccida. Mol Ecol 15:2305-2315 